\title{
Scoliosis and sagittal balance in Parkinson's Disease: analysis of correlations
}

\author{
Luciano Bissolotti $^{1 *}$, Sabrina Donzelli ${ }^{2}$, Massimiliano Gobbo ${ }^{3}$, Fabio Zaina ${ }^{2}$, Jorge Hugo Villafane ${ }^{4}$, Stefano Negrini ${ }^{5}$ \\ From 11th International Conference on Conservative Management of Spinal Deformities - SOSORT 2014 \\ Annual Meeting \\ Wiesbaden, Germany. 8-10 May 2014
}

\section{Background information}

The knowledge concerning scoliosis in Parkinson's Disease (PD) and its correlations with sagittal balance (SB) is sparse.

\section{Purpose}

The aim of this study was to describe the prevalence of scoliosis in PD patients and the existing correlations with $\mathrm{SB}$ in relation to the spinopelvic morphology.

\section{Methods}

64 consecutive PD patients were included: 53 males, 11 females; $69.5 \pm 8.1$ years; $5.6 \pm 4.1$ years of disease (YOD); Hoehn Yahr (H\&Y) 2.4 \pm 1.1 , UPDRS-M 16.1 \pm 12.5 . The clinical assessment included HY and UPDRS-M score, Pain NRS 0-10 and trunk rotation in bending (ATR). Lumbar lordosis (LL), thoracic kyphosis (TK), scoliosis curves (SC), spinosacral angle (SSA), spinopelvic angle (SPA), pelvic incidence (PI), sacral slope (SS) and pelvic tilt (PT) were radiographically assessed. Patients have been compared according to the presence of SC $>10^{\circ}$ (PDts) Cobb or the absence of SC (PDns).

\section{Results}

$45 \%$ of cohort presented a SC larger than $11^{\circ}, 84 \%$ of the patients in PDts presented a thoracolumbar curve, $10 \%$ a thoracic one and $6 \%$ a lumbar one. They did not present differences with PDns about age $(70.8 \pm 6.8$ vs $68.8 \pm 8.6 \mathrm{yrs})$ and YOD $(6.6 \pm 6.0$ vs $6.1 \pm 4.3 \mathrm{yrs})$. No differences have been detected for H\&Y score $(2.6 \pm 1.2$ vs 2.6 $\pm 1.1)$ and UPDRS-M $(16.5 \pm 12.3$ vs $16.8 \pm 12.4)$. Pain was slightly higher in PDts than PDns (3.2 \pm 2.7 vs $2.3 \pm 2.8)$. ATR was higher in PDts $(5.0 \pm 4.3$ vs $1.5 \pm 2.0, \mathrm{p}<0.01)$.
TK $\left(47.1 \pm 14.5\right.$ vs $\left.45.5 \pm 12.5^{\circ}\right)$, LL $(47.5 \pm 24.2$ vs 50.3 $\left.\pm 13.9^{\circ}\right)$, SSA $\left(111.7 \pm 22.3\right.$ vs $\left.120.1 \pm 10.9^{\circ}\right)$ and SPA $(154.4$ \pm 18.2 vs $\left.156.9 \pm 12.3^{\circ}\right)$ were not different $(\mathrm{p}>0.05)$. PI $\left(55.3 \pm 11.2\right.$ vs $\left.53.6 \pm 11.4^{\circ}\right)$ and PT $(21.6 \pm 13.2$ vs 16.8 $\pm 8.1^{\circ}$ ) were slightly but not statistically different, while SS was not $\left(34.7 \pm 11.2\right.$ vs $\left.36.2 \pm 9.3^{\circ}\right)$.

\section{Conclusions and discussion}

Pelvic parameters did not differ significantly in the two groups. The prevalence of scoliosis in PD was higher than previously described by the other Authors and thoracolumbar spine was the mostly affected. SB was not different between two groups while, in PDts, spinopelvic parameters presented the tendency to have a larger PT. Further studies will deepen this issues, in PD patients with more severe scoliosis.

\section{Authors' details}

${ }^{1}$ Rehabilitation Service, Fondazione Teresa Camplani Casa di Cura Domus Salutis, Brescia, Italy. ${ }^{2}$ ISICO, Milan, Italy. ${ }^{3}$ Department of Clinical and Experimental Sciences, University of Brescia, Brescia, Italy. ${ }^{4}$ IRCCS Fondazione Don Gnocchi ONLUS, Milan, Italy. ${ }^{5}$ RCCS Fondazione Don Gnocchi ONLUS, Milan, Italy, ISICO, Milan, Italy.

Published: 4 December 2014

doi:10.1186/1748-7161-9-S1-038

Cite this article as: Bissolotti et al: Scoliosis and sagittal balance in Parkinson's Disease: analysis of correlations. Scoliosis 2014 9(Suppl 1):O38. 